\title{
Calidad asistencial y satisfacción de las mujeres a tratamiento por drogodependencia en Asturias.
}

\author{
Cristina Arnáez Montaraz(1); Pedro Alberto Marina González ${ }^{(2)}$; \\ Roberto Secades Villa ${ }^{(3)}$; José Ramón Fernández Hermida ${ }^{(3)}$. \\ Psicóloga. Colegio Oficial de Psicólogos del Principado de Asturias (1) \\ Psiquiatra. Unidad de Tratamiento de Toxicomanías de Oviedo. Servicios de Salud Mental del Principado de Asturias (2) \\ Psicólogo. Profesor Titular de Psicología. Grupo de Conductas Adictivas. Departamento de Psicología. Universidad de Oviedo (3) \\ Enviar correspondencia a: \\ Pedro A. Marina González. Unidad Tratamiento Toxicomanías Hospital General. C/ Julián Clavería s/n. 33006 Oviedo (Asturias) \\ Teléfono: 985106 156. Fax: 985106 157. e-mail: pepaz@arrakis.es.
}

Recibido: 4 de septiembre de 2003 Aceptado:: 13 de abril de 2004

\section{RESUMEN}

En los últimos años se ha enfatizado la importancia de la valoración de la calidad de los servicios de salud por parte de los propios pacientes. Esta valoración es útil para orientar a los responsables de las instituciones de cara a mejorar los recursos asistenciales. El objetivo de este estudio es evaluar el grado de satisfacción de las mujeres con los tratamientos de drogodependencias en el Principado de Asturias. La investigación se llevó a cabo con una muestra de 108 mujeres que estaban a tratamiento en alguno de los dispositivos públicos o concertados de Asturias. Las entrevistas fueron realizadas "cara a cara" o a través del teléfono. Los resultados mostraron que el nivel de satisfacción de las usuarias con respecto a los programas de tratamiento para drogodependientes en el Principado de Asturias era elevado. Asimismo, la valoración que realizan las mujeres sobre los profesionales de los diferentes dispositivos asistenciales es alta. Los centros privados-concertados obtienen una valoración superior en comparación con los centros públicos.

Palabras clave: Calidad, satisfacción, mujeres, tratamientos de drogodependencias.

\section{ABSTRACT}

The importance of the patients' opinion on the quality of health services has been emphasized in the last few years. This evaluation is useful in guiding those responsible for the centres in improving welfare resources. The present study was designed to evaluate the satisfaction level of drug addicted women with the treatment they received in Asturias (Spain). The research was carried out with a sample of 108 drug-addicted women who were undergoing treatment in public or private centres in Asturias. The interviews were "face to face" or by telephone. The results showed a high satisfaction level among drug-addicted women who had followed treatment programmes in Asturias. In addition, the personnel working in the different welfare centres were evaluated most highly by these women. Private centres score higher than public centres.

Key words: quality, satisfaction, women, drug abuse treatment

\section{INTRODUCCIÓN}

L

os estudios para conocer la opinión de los usuarios acerca de las prestaciones socio-sanitarias

que reciben son relativamente recientes y obedecen al interés por medir la satisfacción de los clientes según un modelo de gestión que tiene como eje la calidad. Este tipo de estudios ya son habituales en la atención primaria de salud y cada vez más frecuentes en los hospitales y otros contextos asistenciales, incluyendo también el ámbito de las adicciones.
La calidad es entendida como un instrumento de gestión que pretende conseguir una organización más efectiva y eficiente, que se ajuste a las necesidades de los clientes y de la comunidad. Desde este punto de vista, la satisfacción de los usuarios se constituye como uno de los elementos más importantes. Por eso, todo sistema de gestión en calidad incluye, junto a otros, el estudio de la calidad percibida como manera de conocer la opinión, satisfacción y expectativas de los clientes. La opinión de los pacientes, en el caso de los servicios de salud, es útil para conocer la imagen que éstos tienen de dichos servicios y para desarrollar 
estrategias orientadas a incrementar la satisfacción de los usuarios.

La época en la que los usuarios de los servicios de salud eran sujetos pasivos, meros pacientes receptores de diferentes técnicas diagnósticas o terapéuticas cuya complejidad rebasaba su capacidad de comprensión, ha quedado atrás. Ahora estamos en la era del paciente (Reiser, 1993), en la que el punto de vista de éste es esencial para tomar las decisiones que competen a su salud y también para la planificación y organización de los servicios.

Se han realizado muchos estudios que han intentado desentrañar los componentes de la calidad percibida, ordenar su importancia y conocer los factores que condicionan la percepción de calidad de los usuarios (Donabedian, 1984; Cottle, 1991; Hayes, 1995). En este sentido, hay acuerdo en que las expectativas de los usuarios con respecto al servicio juegan un papel fundamental en la calidad percibida, de modo que ésta será mayor o menor según el nivel de concordancia o discrepancia entre las expectativas y el servicio recibido.

Así, la medida de la satisfacción ha demostrado ser un instrumento útil para evaluar las intervenciones de los servicios sanitarios. El objetivo del análisis de la satisfacción es facilitar información a profesionales y gestores del sistema sanitario acerca de aquellos aspectos que son percibidos como insatisfactorios y son susceptibles de mejora (Caminal, 2001).

La medida de la satisfacción se realiza generalmente mediante encuestas con una metodología que combina técnicas cualitativas y cuantitativas. En este sentido, se han desarrollado algunos instrumentos de medida ampliamente validados, como es el caso de los cuestionarios derivados del SERVQAL para su uso en el ámbito hospitalario o en Atención Primaria (Bretones y Sánchez, 1989).

En el ámbito del consumo de drogas han sido pocos los estudios realizados con el fin de valorar la calidad de los programas asistenciales y la satisfacción de los usuarios de los mismos. Solo en fechas recientes se han empezado a utilizar nuevas herramientas como el "Maudsley Addiction Profile" (MAP, ERIT-Versión), el "Treatment Perceptions Questionnaire" (TPQ) o la Escala de Verona como instrumentos rápidos para la investigación con poblaciones con desórdenes derivados del consumo de drogas (Marsden et al, 2001; De los Cobos et al, 2002).

En nuestro país, Rodríguez et al. (2002) realizaron un estudio en el que evaluaron la satisfacción con el tratamiento en un centro ambulatorio de drogodependencias a través del TPQ. Los resultados mostraron que los usuarios estaban satisfechos tanto con el programa como con el equipo terapéutico.
Haro et al. (2002) evaluaron el nivel de satisfacción de los usuarios de la red de drogodependencias de la comunidad valenciana en relación con el conocimiento de sus derechos como pacientes. Los resultados mostraron que la mayor parte de los pacientes estaban satisfechos con los servicios prestados en la UDH y en su UCA, encontrándose pocas diferencias en la satisfacción entre estos servicios.

De la misma manera, han sido muy escasos los estudios realizados para analizar las características particulares de la población drogodependiente femenina En esta línea, el colectivo de mujeres drogodependientes es de los que presentan una mayor necesidad de estudios de esta naturaleza puesto que el entorno asistencial en el que se mueven fue diseñado en respuesta a las demandas de la mayoría masculina, presentando ellas necesidades diferentes y en ciertos aspectos especiales (Reed, 1987, Stocco, 1998). Pocos han sido los estudios que compararan diferencias de género. Briñez Horta (2001) evaluó las diferencias en la Intoxicación, Abuso y Dependencia del alcohol, entre hombres y mujeres, en una muestra de 910 estudiantes universitarios. Los resultados mostraron que los hombres tienen mayor riesgo de intoxicación, de abuso y de dependencia alcohólica. Las mujeres presentan mayor riesgo de manifestar estos mismos problemas bajo el consumo severo. Estos problemas se incrementan progresivamente en relación directa con el nivel de consumo, en ambos géneros. Además, las mujeres presentan mayor riesgo de signos fisiológicos de intoxicación y los hombres mayor riesgo de pérdida del autocontrol.

En esta coyuntura, se echan de menos en nuestro medio estudios que contribuyan a conocer la valoración que las personas con problemas de alcohol y drogas hacen de las distintas modalidades de tratamiento y la adecuación de estos a sus necesidades y expectativas, sobre manera cuando los estudios realizados en otros países muestran que los programas de tratamiento que atienden a las necesidades de sus pacientes obtienen mejores resultados (Nelson et al, 1996; McLellan y Hunkeler, 1998; Hser et al, 1999; Conners y Franklin, 2000).

El estudio que aquí se presenta se enmarca dentro de esta línea de trabajo. El objetivo fundamental del mismo es evaluar el grado de satisfacción que tienen las mujeres con los tratamientos de drogodependencias en el Principado de Asturias. Además, se pretende también analizar y conocer:

- El perfil de la población femenina que es atendida en los centros de atención a drogodependientes de esta Comunidad.

- El nivel de satisfacción de las mujeres con los tratamientos recibidos en los distintos recursos asistenciales. 
- La valoración de las mujeres toxicómanas acerca de la accesibilidad a los recursos de atención a drogodependientes.

- El nivel de adecuación de los recursos asistenciales con relación con las necesidades específicas de la mujer.

- Las demandas y necesidades específicas de las mujeres en los distintos tratamientos de deshabituación de drogas en Asturias.

\section{MATERIALY MÉTODOS}

\section{Muestra}

La investigación se llevó a cabo con la totalidad de mujeres que estaba a tratamiento por dependencia de sustancias psicoactivas en alguno de los dispositivos públicos o concertados de Asturias y que, además, habían sido atendidas en los últimos seis meses del año 2000. En concreto, los criterios de inclusión de las participantes en el estudio fueron los siguientes: ser mujer, haber sido diagnosticada de trastorno por dependencia de sustancias psicoactivas según criterios de la CIE-10 (OMS, 1992), haber sido atendidas entre julio y diciembre del 2000 por dependencia de sustancias psicoactivas en cualquiera de los recursos públicos y/o concertados (Centros de Salud Mental -CSMUnidades de Desintoxicación Hospitalaria -UDH-, Unidades de Tratamiento de Toxicomanías, encargadas de los tratamientos de sustitución con metadona -UTT-, Proyecto Hombre y Centro Spiral) del Principado de Asturias, y estar a tratamiento en el momento de realización del estudio en el mismo recurso asistencial o en otro al que hayan sido derivadas.

La muestra final se compuso de 108 mujeres con una edad media de 33,37 años ( $s=9,64$ y rango de 17 a 60 años), que llevaban a tratamiento una media de 28,5 días. Algunas características sociodemográficas de la muestra aparecen en la Tabla 1.

Tabla 1. Características sociodemográficas de la muestra

\begin{tabular}{|lllc|}
\hline Edad & Media & & 33,37 años \\
& Rango & $17-60$ & \\
\hline Estado Civil & Soltera & & $49,1 \%$ \\
& Casada & $26,9 \%$ & \\
& Separada & $13,0 \%$ & \\
& Divorciada & $8,3 \%$ & \\
& Viuda & $2,8 \%$ & \\
\hline Estudios & Sin estudios & $12 \%$ & \\
& Primarios & & \\
& Secundarios & $26,9 \%$ & \\
& Universitarios & $10,2 \%$ & \\
& Ns/Nc & $0,9 \%$ & \\
\hline Pareja en la actualidad & Sí & $64,8 \%$ & \\
& No & $35,2 \%$ & \\
\hline
\end{tabular}

La distribución de las participantes en los distintos centros de tratamiento fue la siguiente: Centros de Salud Mental (17,6\%), Unidades de Metadona (37,04\%), Unidades de Desintoxicación Hospitalaria (12,03\%), Proyecto Hombre (24,07\%), Centro Spiral (9,25\%).

\section{Variables e instrumentos}

El instrumento de recogida de información fue un cuestionario heteroaplicado elaborado "ad hoc" para la investigación que constaba de 65 preguntas que recogían información acerca de los siguientes apartados: datos descriptivos, accesibilidad, variables relaciona- das con la condición de mujer, tipos de intervención, información, valoración de los profesionales, estructura del programa, satisfacción y necesidades percibidas.

\section{Procedimiento}

En un primer momento se realizó un estudio piloto para comprobar la idoneidad del cuestionario, pasando el mismo a una muestra de mujeres que cumplían los criterios de inclusión en el estudio. Una vez confirmada la fiabilidad y operatividad del instrumento de medición, se procedió a la recogida definitiva de datos, prolongándose ésta durante 9 meses. Las entrevistas 
fueron realizadas por psicólogos previamente entrenados en estas tareas, una vez que la Consejería de Salud y Servicios Sanitarios solicitó la colaboración en el estudio de los distintos recursos seleccionados. Las entrevistas fueron realizadas cara a cara (38\%) o telefónicamente (62\%).

\section{Análisis de datos}

Los procedimientos estadísticos realizados fueron el análisis de frecuencias, análisis bivariados y el ANOVA. El paquete estadístico utilizado fue el SPSS+, versión 11.0 .

\section{RESULTADOS}

\section{Perfil de las participantes}

En las tablas 2 y 3 se muestran los datos acerca de las características médicas-judiciales y sobre la historia de uso de drogas respectivamente.

\section{Nivel de satisfacción de las mujeres con el tra- tamiento recibido}

El nivel de satisfacción de las mujeres entrevistadas con el tratamiento es elevado. El 58,3\% manifiesta que está satisfecha y el $27,8 \%$ muy satisfecha con

\section{Tabla 2. Variables médicas y judiciales}

\begin{tabular}{|llc|}
\hline Problemas médicos crónicos & Sí & $51,9 \%$ \\
& No & $48,1 \%$ \\
\hline Problemas psiquiátricos & Sí & $61,1 \%$ \\
& No & $38,9 \%$ \\
\hline Tipo de problema psiquiátrico & Depresión & $71,87 \%$ \\
& Ansiedad & $10,94 \%$ \\
& Depresión-Ansiedad & $6,25 \%$ \\
& Trastornos de la alimentación (anorexia) & $4,68 \%$ \\
& Otros & $6,25 \%$ \\
\hline Número de detenciones & Media & 1,71 \\
& Rango & $0-40$ \\
\hline Número de ingresos en prisión & Media & 0,45 \\
& Rango & $0-6$ \\
\hline Número de juicios pendientes & Media & 0,13 \\
& Rango & $0-2$ \\
\hline
\end{tabular}

Tabla 3. Características del consumo de drogas de las participantes

\begin{tabular}{|llc|}
\hline Droga principal de consumo & Heroína & $68,5 \%$ \\
& Cocaína & $6,5 \%$ \\
& Alcohol & $22,2 \%$ \\
& Otras & $2,8 \%$ \\
\hline Años de consumo de la droga principal & Media & 7,81 \\
& Rango & $0-40$ \\
\hline Vía de administración droga principal & Oral & $23,1 \%$ \\
& Nasal & $1,9 \%$ \\
& Fumada & $41,7 \%$ \\
& Intravenosa & $14,8 \%$ \\
& Fumada e intravenosa & $16,7 \%$ \\
& Otras & $1,9 \%$ \\
\hline Tratamientos anteriores con ayuda profesional & Media & 1,97 \\
& Rango & $0-20$ \\
\hline Tiempo en días en el tratamiento actual & Media & 28,45 \\
& Rango & $3-2555$ \\
\hline Tiempo en días abstinencia tratamiento actual & Media & 290,23 \\
& Rango & $0-2555$ \\
\hline Período máximo de abstinencia en días & Media & 508,86 \\
& Rango & $0-2555$ \\
\hline Número de sobredosis con asistencia & Media & 0,65 \\
& Rango & $0-10$ \\
\hline
\end{tabular}


el tratamiento que está recibiendo en ese momento. Sólo el 3,7\% de las mujeres entrevistadas responde que no está satisfecha con el tratamiento. Estos datos son concordantes con las respuestas a la pregunta: ¿recomendaría el tratamiento a un amigo/a con problemas de adicción?, en la cual, el 73,1\% responde que está muy de acuerdo, el 14,8\% de acuerdo, mientras que el 1,9\% está en desacuerdo y el 3,7\% muy en desacuerdo.

En cuanto al nivel de satisfacción en función de los distintos dispositivos asistenciales, los centros privados son los dispositivos mejor valorados, seguidos de las UDH, las Unidades de Metadona (UTT) y los CSM.

Los análisis estadísticos mostraron la existencia de diferencias estadísticamente significativas ( $p<0.05$ ) entre los centros públicos y los privados concertados, en las preguntas: ¿está satisfecha con la atención prestada en este tratamiento? y ¿recomendaría este tratamiento a algún amigo/a con problemas de adicción?. Los centros de privados fueron mejor valorados en ambas preguntas. La valoración de los diferentes dispositivos asistenciales de más a menos es la siguiente: Proyecto Hombre, Spiral, Unidades de Desintoxicación Hospitalaria, Unidades de Metadona y Centros de Salud Mental (Tabla 4). se resolvió eficazmente, mientras que el 3,7\% afirma que no se solucionó.

En cuanto a las expectativas sobre el tratamiento, el 70,4\% de las mujeres encuestadas está de acuerdo en que el tratamiento se ajustó a lo que esperaba encontrar en él. Sólo el 6,5\% contestó que el tratamiento no había respondido a las expectativas previas al ingreso en éste.

Un factor muy importante con relación a la satisfacción de las usuarias drogodependientes con los programas de tratamiento es la valoración que éstas realizan de los profesionales que atienden los distintos dispositivos asistenciales. La mayoría de las mujeres encuestadas (de acuerdo: 64,8\% o muy de acuerdo: $21,3 \%$ ) afirman que los profesionales de los diferentes programas las escuchan y las ayudan convenientemente. Además, opinan que la relación que establecen los profesionales con ellas es buena en un $75 \%$ de los casos y excepcional en el 15,7\%. También afirman en un $67,6 \%$ que los profesionales comprenden las necesidades que ellas tienen.

El $74,1 \%$ de las entrevistadas esta de acuerdo o muy de acuerdo $(16,7 \%)$ en que los profesionales del equipo técnico que las atienden posee un buen nivel de competencia profesional para ayudarlas eficazmente en su problema de drogadicción.

\section{Tabla 4. Satisfacción con el tratamiento en los distintos dispositivos evaluados}

\begin{tabular}{|c|c|c|c|c|c|c|}
\hline & \multicolumn{2}{|c|}{$\begin{array}{l}\text { Estoy satisfecha con la } \\
\text { atención que me han prestado }\end{array}$} & \multicolumn{2}{|c|}{$\begin{array}{l}\text { El tratamiento se ajustó a } \\
\text { lo que se esperaba de él }\end{array}$} & \multicolumn{2}{|c|}{$\begin{array}{c}\text { Recomendaría el tratamiento } \\
\text { a un amigo/a }\end{array}$} \\
\hline & Media & $\sigma$ & Media & $\sigma$ & Media & $\sigma$ \\
\hline CSM & 2.16 & 1.90 & 2.53 & 0.90 & 1.68 & 1.29 \\
\hline U. de metadona & 2.05 & 0.50 & 2.48 & 1.24 & 1.85 & 1.36 \\
\hline $\mathrm{PH}$ & 1.54 & 0.58 & 2.08 & 1.09 & 1.07 & 0.39 \\
\hline SPIRAL & 1.80 & 0.92 & 2.40 & 1.26 & 1.30 & 0.67 \\
\hline $\mathrm{UDH}$ & 1.85 & 0.90 & 2.77 & 1.59 & 1.46 & 1.12 \\
\hline \multirow[t]{2}{*}{ Total } & 1.90 & 0.72 & 2.42 & 1.20 & 1.53 & 1.13 \\
\hline & \multicolumn{2}{|c|}{$\begin{array}{l}\text { 1: muy satisfecha } \\
\text { 5: muy insatisfecha }\end{array}$} & \multicolumn{2}{|c|}{$\begin{array}{l}\text { 1: Muy de acuerdo } \\
\text { 4: Muy en desacuerdo }\end{array}$} & \multicolumn{2}{|c|}{$\begin{array}{l}\text { 1: Sí, con toda seguridad } \\
\text { 5: No, con toda seguridad }\end{array}$} \\
\hline
\end{tabular}

En los centros dependientes de Salud Mental (CSM y UTT) un 81,4\% están satisfechas o muy satisfechas con la atención prestada durante el tratamiento. En la UDH, el porcentaje es del $84,7 \%$ y en los centros privados el $94,4 \%$.

A la pregunta de si han tenido algún problema con los servicios prestados en el tratamiento, el 86,1\% de las encuestadas afirmaban no haber tenido ningún tipo de problema. Del 11,1\% que decían que habían tenido uno o más problemas en el recurso en el que se encontraban, el 7,4\% manifiesta que ese problema
En cuanto a la rapidez con la que los profesionales atienden a las demandas planteadas por las usuarias del tratamiento, el 78,7\% de las entrevistadas responden que es alta. Solo el 3,7\% opina que la respuesta a sus demandas y peticiones es lenta.

\section{Accesibilidad}

En general, las mujeres entrevistadas valoran la accesibilidad a los programas de tratamiento positivamente. 
El acceso al tratamiento se considera fácil en el $72,2 \%$ de las ocasiones y muy fácil en el 12,1\%. La mayor dificultad externa para acceder al tratamiento son las listas de espera (13,9\%), si bien un $42,6 \%$ manifiesta que no existe ninguna dificultad a la hora de acceder al tratamiento.

El motivo fundamental de elección del tratamiento en el que se encuentra la paciente es en la mayoría de los casos, la recomendación de familiares y amigos $(29,6 \%)$, seguido de la recomendación de algún profesional $(27,8 \%)$.

\section{Adecuación de los recursos asistenciales}

El 98,1\% de las mujeres entrevistadas piensa que el hecho de ser mujer no conlleva diferencias en el trato por parte de los profesionales. Tan solo un 1,9\% opina que el ser mujer ocasiona que algunas veces los profesionales las traten peor. También piensan que los usuarios masculinos del programa las tratan igual en un $74,1 \%$. El hecho de ser mujer no parece traer ni ventajas ni desventajas a la hora de realizar el tratamiento en un $90,7 \%$ de las ocasiones. En todo caso, el hecho de ser mujer supone más una ventaja (4,6\%) que una desventaja $(0,9 \%)$.

A la hora de establecer un vínculo terapéutico, el $28,7 \%$ de las mujeres prefieren hacerlo con otra mujer, mientras que un $8,3 \%$ prefieren un hombre. Sin embargo, la mayoría (un 61,1\%) manifiesta no tener preferencias de cara al vínculo profesional.

\section{Demandas y necesidades}

El 56,5\% de las mujeres afirmaba que no había ningún tipo de atención que echase en falta en el tratamiento. Sin embargo, un porcentaje importante de usuarias $(38,2 \%)$ si echaba en falta algún recurso o servicio específico en el programa de tratamiento. El servicio más demandado, con un $15,7 \%$, es la atención psicológica. Por el contrario, la atención médica se encuentra suficientemente cubierta según la opinión de las mujeres entrevistadas. Por otra parte, el $70,4 \%$ esta de acuerdo con que el tratamiento le ofrece una atención individualizada, atendiendo a sus necesidades particulares.

En cuanto al papel de la familia, el 51,9\% de las usuarias entrevistadas afirma que su familia interviene en el tratamiento que está recibiendo. El 34,3\% responde que su familia no interviene en el tratamiento.

Sobre el número de profesionales en los diferentes programas, el $58 \%$ de las mujeres están de acuerdo en que es suficiente, mientras que un 15, 7\% opina que el número de profesionales implicados en su tratamiento es insuficiente.

Por lo que se refiere a las instalaciones, el 72,2\% de las pacientes cree que las instalaciones del recurso en el que se encuentran a tratamiento son adecuadas.
Un porcentaje muy pequeño $(4,6 \%)$ no está de acuerdo con esta afirmación.

\section{DISCUSIÓNY CONCLUSIONES}

El objetivo general de esta investigación era evaluar el grado de satisfacción que tienen las mujeres usuarias de los dispositivos asistenciales de drogodependencias del Principado de Asturias, aunque permitió, asimismo, conocer el perfil de la población femenina con trastornos adictivos.

En este sentido, más de la mitad de la muestra solo tiene estudios primarios o no posee ningún tipo de certificación académica. Alrededor del 42\% está trabajando antes de iniciar el tratamiento, si bien el porcentaje de mujeres en activo desciende en el momento del tratamiento. La inserción de la mujer en el mundo laboral es un objetivo importante que se debería de contemplar al menos en las fases más avanzadas de los programas de deshabituación. Así mismo, la baja cualificación laboral encontrada en las mujeres participantes hace fundamental la incorporación de éstas a planes formativos que puedan facilitar posteriormente la consecución de un empleo.

El porcentaje de problemas médicos crónicos en la muestra de mujeres es similar al encontrado en hombres en otros estudios revisados (Fernández, 2002). Aunque los estudios epidemiológicos muestran que la comorbilidad de trastornos por uso de sustancias y otros trastornos mentales es muy elevada (GarcíaCampayo y Sanz, 2002) llama la atención el elevado porcentaje de consultas por problemas psiquiátricos, en particular por depresión que aparece como el trastorno de mayor prevalencia. Estos datos señalan la importancia de ofrecer intervenciones específicas, dentro de los tratamientos de deshabituación, encaminadas a disminuir la incidencia de trastornos psicopatológicos en este tipo de pacientes.

Con respecto a los hombres, las mujeres tienen un historial delictivo menor: presentan un menor número de detenciones, ingresos en prisión y juicios pendientes.

Las características y el patrón de consumo no parecen mostrar diferencias reseñables con respecto a los hombres de otros estudios de nuestro medio en las variables años de consumo y vía de administración (Gutiérrez y col., 1998; Fernández, 2002). Sin embargo las mujeres presentaban menor número de episodios de sobredosis y menos tratamientos anteriores con ayuda profesional. Así pues, a pesar de que la severidad de la adicción es similar, las mujeres acceden menos a los tratamientos de deshabituación ya sean públicos o privados. 
Los motivos para iniciar el tratamiento tienen que ver con la iniciativa propia en el $74,1 \%$ de los casos. La presión familiar influye en la decisión para iniciar el tratamiento en el $24,1 \%$ de los casos. Otras razones como la indicación profesional, el deterioro físico, el mandato judicial o la presión de la pareja no parecen ser tan importantes. Este dato parece indicar que el menor acceso de la mujer a los programas de tratamiento se debe más a características personales que a la dificultad objetiva para acceder a los recursos.

Por lo que se refiere al objetivo principal de este trabajo, los resultados obtenidos muestran que el nivel de satisfacción general de las usuarias con respecto a los programas de tratamiento que se han estudiado es elevado. Esta opinión mayoritariamente favorable se ve reflejada en los diferentes apartados de la entrevista.

La valoración que realizan las mujeres encuestadas de los profesionales que trabajan en los diferentes dispositivos asistenciales es muy alta. Se valora positivamente tanto la relación, como la atención y la capacitación de los profesionales. También refieren una buena o excepcional relación con los profesionales que las atienden. Además, éstos son vistos como competentes y con capacidad para ayudarlas eficazmente a resolver sus problemas.

Los dispositivos asistenciales mejor valorados son los privados, seguidos de Unidad de Desintoxicación Hospitalaria, Unidades de Metadona y Centros de Salud Mental. Sería de interés indagar las razones que explican estas diferencias y, en particular, sería importante conocer las causas particulares por las que las mujeres entrevistadas se encuentran menos satisfechas con los centros públicos. Una de las razones que puede influir en esta mejor valoración de los centros privados, es que éstos cuentan con intervenciones específicas destinadas a cubrir las necesidades y demandas particulares que plantean las mujeres, en la línea apuntada por Reed (1987)

Algunos estudios muestran que los usuarios de los recursos de salud tienden a valorar positivamente los servicios recibidos, con niveles de satisfacción altos que varían entre el $75 \%$ y el $98 \%$. Lehman y Zastowny (1983) en una revisión de estudios de satisfacción con los servicios de salud mental señalan un nivel global de satisfacción del 70,6\%. En nuestra muestra el grado de satisfacción es significativamente superior, si bien desciende en aspectos concretos del tratamiento, pero, al mismo tiempo es muy similar al encontrado en la última encuesta de satisfacción de usuarios con los servicios de atención primaria del INSALUD (1999).

La accesibilidad a los recursos es buena. Sin embargo, es relevante el hecho de que el 13,9\% han tenido problemas con las listas de espera. No cabe duda que la atención inmediata es un requisito importante en todos los tratamientos y más en el caso de las personas adictas a drogas, por lo que sería deseable minimizar en la medida de lo posible las demoras a la hora de iniciar los tratamientos.

El análisis de las variables relativas a la condición de mujer dentro del tratamiento mostró que las mujeres no se sentían tratadas de manera diferente por cuestión de género. Además, en el caso de haber algún tipo de "discriminación", ésta sería positiva, ya que las entrevistadas opinan que el hecho de ser mujeres les traía dentro del tratamiento más ventajas que inconvenientes.

Más de la mitad de las mujeres encuestadas opinan que el tratamiento que están realizando posee todas las atenciones necesarias para su rehabilitación. Sin embargo un $15,7 \%$ de los casos demandan más atención psicológica. Esta petición puede estar relacionada con los trastornos depresivos que muchas usuarias parecen presentar.

La atención integral en la que se tengan en cuenta las múltiples necesidades de los adictos a las drogas es un principio puesto de relieve por los organismos e instituciones internacionales más importantes (NIDA, 1999). Así, sería conveniente incrementan el peso de la atención psicoeducativa, sobre todo, en determinados dispositivos asistenciales en los que este tipo de recurso es totalmente insuficiente.

El nivel de información que tienen las mujeres sobre los recursos asistenciales para drogodependientes es medio. Un porcentaje importante de mujeres $(26,9 \%)$ manifestaba que no está suficientemente informada sobre lugares donde acudir para recibir ayuda.

El grado de satisfacción con respecto a determinadas variables estructurales, aún siendo alto, desciende en comparación con los ítems anteriores. En cuanto al número de profesionales implicados en el tratamiento, un porcentaje considerablemente elevado de mujeres cree que el número de profesionales que forman el equipo terapéutico es insuficiente. Aumentar el número de profesionales en los tratamientos o disminuir el volumen de personas por equipo podría ser una alternativa para conseguir mejorar la satisfacción.

En función de los datos anteriores, podemos afirmar que los recursos asistenciales de atención a drogodependencias del Principado de Asturias responden adecuadamente a las necesidades y expectativas de las mujeres. Sin embargo, el hecho de que el nivel de satisfacción de las mujeres en los tratamientos sea elevado no implica que las usuarias no tengan demandas que facilitarían una satisfacción aún más alta. De hecho, más de un $60 \%$ de las mujeres aportan peticiones de cara a mejorar los recursos en los que están siendo tratadas. Las demandas que hacen correlacionan con aquellos aspectos del tratamiento en los que 
la satisfacción era más baja. En cuanto a infraestructura, las mujeres demandan, en general, una mejora de las instalaciones y un aumento del número de profesionales que intervienen en el tratamiento. Se observa que muchas usuarias desconocen la categoría del profesional o de los profesionales que intervienen de manera más directa en su rehabilitación. Por ello, cuando se demandan más psicólogos, psiquiatras o incluso médicos, quizás se está solicitando una atención más integral en todos los planos de la persona.

Se resalta también las necesidades en el ámbito de la formación/inserción laboral, solicitando talleres y cursos ocupacionales que puedan mejorar su cualificación profesional.

Otra demanda específica es la inmediatez y la duración de la atención. La demora a la hora de materializar las consultas y la escasez de duración de cada una de ellas se presentan como un factor a mejorar en las valoraciones realizadas por las mujeres.

Los resultados de esta investigación no hacen más que describir el panorama general de la satisfacción y la calidad percibida de los recursos asistenciales en drogodependencias en el Principado de Asturias. Por tanto, este estudio se debería entender como una primera aproximación o como el inicio de una línea de trabajo que tenga como argumento esencial analizar de manera más exhaustiva, específica y continuada los procesos de los tratamientos que sean susceptibles de ser mejorados. Sin duda, la opinión de los usuarios de estos dispositivos es una fuente de información muy valiosa de cara a la toma de decisiones con respecto a la red pública y privada de salud.

\section{Este estudio ha sido financiado por la Conseje- ría de Salud y Servicios Sanitarios del Principado de Asturias (Plan Regional sobre Drogas).}

Los autores agradecen al personal de los Centros donde se realizó el estudio la colaboración prestada.

\section{REFERENCIAS}

Bretones, C., Sánchez, J.E. (1989). Construcción y validación de una encuesta de satisfacción de usuarios de atención primaria. Atención Primaria, 6, 5, 312-316.

Briñez-Horta, J.A. (2001). Diferencias de género en problemas con el alcohol, según nivel de consumo. Adicciones, 13, Número 4.

Caminal, J. (2001). La medida de la satisfacción: un instrumento de participación de la población en la mejora de la calidad de los servicios sanitarios. Revista Calidad Asistencial 16, 276-279.
Conners, N.A., Franklin, K.K. (2000). Using focus groups to evaluate client satisfaction in an alcohol and drug treatment program. Journal of Substance Abuse Treatment, 18, 4, 313-320.

Cottle, D. (1991). El servicio centrado en el cliente. Madrid: Editorial Díaz de Santos.

De los Cobos, J., Valero, S., Haro, G., Fidel, G., Escuder, G., Trujols, J., y Valderrama, J.C. (2002). Development and psychometric properties of the Verona Service Satisfaction Scale for methadone-treated opioiddependent patients (VSSS-MT). Drug and Alcohol Dependence, 68, 2, 209-214.

Donabedian, A. (1984). La calidad de la atención médica. México: Editorial Prensa Mexicana.

Fernández Miranda, J.J. (2002). Programas de mantenimiento con metadona: evaluación de su efectividad en Asturias. Oviedo: Servicio de Publicaciones del Principado de Asturias.

García-Campayo, J.; Sanz, C. (2002). Epidemiología de la comorbilidad entre abuso de sustancias y otros trastornos psiquiátricos. En Rubio, López-Muñoz, Álamo y Santo-Domingo editores, Trastornos psiquiátricos y abuso de sustancias. Editorial Médica Panamericana SA. Madrid. 135-154.

Gutiérrez, E.; Sáiz , P.; González, P.; Fernández, J.J.; Bobes, J. (1998). Trastornos de la personalidad en adictos a opiáceos a tratamientos con agonistas vs antagonistas. Adicciones, 10, 2, 121-130.

Haro, G., Fernández-Garcés, M., Pérez-Gálvez, B., Cervera, G., Castellano, M., Valderrama, J.C. y Martínez-Raga J. (2002). Nivel de satisfacción de los usuarios de la red de drogodependencias de la Comunidad Valenciana en relación con el conocimiento de sus derechos como pacientes. Conductas Adictivas, 2, 4.

Hayes BE (1995). Cómo medir la satisfacción del cliente. Barcelona: Ediciones Gestión 2000.

Hser, Y.I., Polinsky, M.L., Maglione, M., y Anglin, M.D. (1999). Matching clients' needs with drug treatment services. Journal of Substance Abuse Treatment. 16, 4, 299-305.

INSALUD (1999). Encuesta de satisfacción de usuarios con los servicios de atención primaria. Resultados 1999. Subdirección General de Atención Primaria. Área de Gestión de Usuarios.

Lehman, A.F., y Zastowny, T.R. (1983). Patient satisfaction with mental health services: a meta-analysis to establish norms. Evaluation and Program Planning, 6, 265-274.

Marsden, J., Nizzoli, U., Corbelli, C., Margaron, H., Torres, M. A., Castro de Prado, I., Stewart, D. y Gossop, M. (2001). Fiabilidad del "Maudsley addiction profile" (MAP, ERITVersion) y del "Treatment perceptions questionnaire" (TPQ) en España, Italia y Portugal para la evaluación de tratamientos. Adicciones, 13, 2, 217-227.

McLellan, A.T. y Hunkeler, E. (1998). Patient satisfaction and outcomes in alcohol and drug abuse treatment. Psychiatry Services. 49, 5, 573-575. 
Nelson-Zlupko, L., Dore, M.M., Kauffman, E. y Kaltenbach, K. (1996). Women in recovery. Their perceptions of treatment effectiveness. Journal of Substance Abuse Treatment, 13, 1, 51-59.

NIDA (1999). Principles of Drug Addiction Treatment. A Research-Based Guide. Rockville: National Institute on drug Abuse (National Institute of Health). Mariland

Organización Mundial de la Salud (1992). ClE-10. Trastornos mentales y del comportamiento. Descripciones clínicas y pautas para el diagnóstico. Ginebra.

Reed, B.G (1987). Developing women sensitive drug dependence treatment services: Why so difficult?. Journal of Psychoactive Drugs, 19,2, 151-164.
Reiser, S.J. (1993). The era of the patient. JAMA, 269, 8, 1012-1017.

Rodríguez, M., Jiménez Lerma, J.M., Iraurgui, I., Murua, F., Bacigalupe, L., Chavarri, M.R. y Balsategui, B. (2002). Evaluación de la satisfacción con el tratamiento en un Centro Ambulatorio de drogodependencias a través del "Treatment Percpetions Questionnaire" (TPQ). Adicciones, 14, 417-424.

Stocco, P. (1998). "Identidad femenina, entre el riesgo y la protección". Adicciones, 10, 2, 107-110 
Arnáez.indd 10 